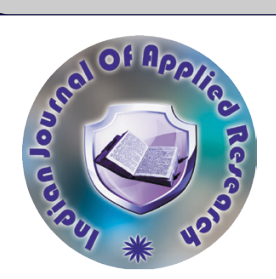

Anesthesiology

\title{
EFFICACY OF ANALGESIA PROVIDED BY ULTRASOUND GUIDED FASCIA ILIACA COMPARTMENT BLOCK WITH AND WITHOUT PARACETAMOL (I.V) IN FACILITATING POSITION FOR CENTRAL NEURAXIAL PROCEDURE
}

\section{Dr. Preethi HN \\ Dr. Pawan Kumar \\ C \\ Dr. Ravishankar \\ BM* \\ ABSTRACT}

Assistant professor, Department of Anesthesiology, Adichunchanagiri Institute of Medical Sciences,BG Nagara, Mandya, Karnataka, India

Junior Resident, Department of Anesthesiology, VIMS, Ballari, Karnataka, India

INTRODUCTION: The use of ultrasound reduces the onset time, improves the quality of sensory block, and minimizes the risks associated with the block. There are few studies supporting the efficacy of ultrasound guided fascia iliaca block METHODOLOGY: 60 patients posted for femur surgeries admitted in Orthopaedics Department, were assessed for the inclusion and exclusion criteria and were included in the study after obtaining written informed consent. RESULTS: Among the patients undergoing spinal anaesthesia in fracture femur surgery, 3.3\% of patients of both the groups had not satisfactory positioning, $36.7 \%$ of Group A and $33.3 \%$ of Group B had satisfactory positioning, $50 \%$ of Group A and $53.4 \%$ of Group B patients had good positioning, $10 \%$ of patients of both groups had optimal positioning. CONCLUSION: Therefore the positioning of the patients for spinal anaesthesia is comparable and is no significant difference is seen among both the groups.

\section{KEYWORDS : Ultrasound Guided Fascia Iliaca Compartment Block, Paracetamol, Central Neuraxial Procedure}

\section{INTRODUCTION:}

Fracture femur is a common orthopaedic problem following trauma and is increasing as the average age of the population increases. Femoral fractures usually cause considerable pain. ${ }^{1}$ Untreated or under-treated pain and potent systemic analgesics can increase the risk of respiratory distress, delirium and other side effects, especially in elderly patients. ${ }^{2}$ The relationship of pain relief to decreased morbidity and mortality remains controversial. The benefits of the acute analgesia play an important role in patient comfort. Although narcotics continue to be the mainstay for pre- and postoperative pain relief, in elderly, avoiding or using very low doses of opioids to treat pain significantly decreases the risk to them of developing delirium ${ }^{3}$.

Central neuraxial blocks such as spinal anaesthesia is the preferred technique for providing anaesthesia. Correct positioning during central neuraxial anesthesia is a prerequisite for successful procedure. However limb immobility and extreme pain are the deterrents for an ideal positioning. Various modalities like femoral nerve block, three in one nerve block, fascia iliaca block, systemic opioids and non opioids, have been advocated to provide analgesia and improve positioning in these patients The administration of a substantial amount of intravenous (i.v.) analgesic is usual practice during spinal block placement. Fascia iliaca block is proposed to provide analgesia and improve positioning in patients with femoral fractures ${ }^{5,6}$.

The use of ultrasound reduces the onset time, improves the quality of sensory block, and minimizes the risks associated with the block There are few studies supporting the efficacy of ultrasound guided fascia iliaca block. We decided to conduct this study with the aim to compare the benefits of block with and without paracetamol (iv) prior to positioning the patient. The quality of positioning for performing central neuraxial procedure will be assessed by ease of performing (no. of attempts, duration of SAB) central neuraxial procedure in patients undergoing surgery for fracture femur ${ }^{7,8}$.

\section{Methodology:}

60 patients posted for femur surgeries admitted in Orthopaedics Department, were assessed for the inclusion and exclusion criteria and were included in the study after obtaining written informed consent.

\section{Sample size:}

The confidence level is estimated at $95 \%$, Standard deviation 3.09 , with a $z$ value of 1.96

The confidence interval or margin of error is estimated at $+/-0.80$

Assuming that 80 percent as power of the study, minimum sample size required for the study was calculated to be 58 .

In our study 60 subjects were chosen
( $\mathrm{n}=30$ in FICB with $0.9 \%$ NS and $\mathrm{n}=30$ in FICB with Paracetamol 1g)

Study Design:

Prospective, Randomized, Double-blind, Controlled study.

\section{Inclusion Criteria:}

1. Patients belonging to ASA grade I and II.

2. Patients of either sex, between the age group 20 to 80 years.

3. Patients with fracture femur, posted for surgery under subarachnoid block

4. Patients who give a valid informed consent.

\section{Exclusion Criteria:}

1. Patients not satisfying inclusion criteria.

2. Patients belonging to ASA grade III or IV.

3. Patients with hemorrhagic diathesis, neurological disorders, psychiatric disorders.

4. Previous femoral bypass surgery.

5. Patients with allergy to local anaesthetics or paracetamol.

6. Patients with polytrauma, infection over the injection site.

7. Patients with liver diseases

8. Morbid obesity.

9. Patients who will be administered with supplementary epidural or general anaesthesia.

10. Patients with spinal deformities.

11. Patients who decline consent

GROUP 1: Patients will receive ultrasound guided fascia iliaca compartment block with $30 \mathrm{ml}$ of $0.5 \%$ Ropivacaine with 30 min prior administration of $100 \mathrm{mlNS} \mathrm{I}$.

All patients with femoral fractures undergoing surgery under central neuraxial anaesthesia will be enrolled for the study.

GROUP 2: patients will receive fascia iliaca block with $30 \mathrm{ml}$ of $0.5 \%$ of Ropivacaine with 30min prior administration of inj Paracetamol $1 \mathrm{~g}$. Central neuraxial anesthesia will be performed in these patients after 30 minutes of giving block in lateral dependent position on the fractured site.

A detailed pre-anaesthetic checkup carried out in each patient. Patient is kept nil per orally 6 hours prior to surgery, i.v line secured, pre medicated with Inj Ranitidine 50mg iv, Inj Ondansetron 4mg iv and IVF Ringer Lactate infusion at $100 \mathrm{ml} / \mathrm{hr} 3$ hours prior to surgery . On arrival to Operation theatre, standard monitors including ECG, Pulse oximeter, and non invasive blood pressure will be attached and baseline vital parameters will be recorded. 
RESULTS:

Table 1: NUMERIC RATING SCALE (NRS) FOR ASSESSMENT OF PAIN

\begin{tabular}{|c|c|c|c|c|c|}
\hline \multirow[t]{2}{*}{ TIME } & \multicolumn{2}{|c|}{ GROUPA } & \multicolumn{2}{|c|}{ GROUP B } & \multirow[t]{2}{*}{ PVALUE } \\
\hline & MEAN & SD & MEAN & SD & \\
\hline Baseline(BL) & 8.1538 & 0.464 & 8.1 & 0.4806 & 0.6729 \\
\hline $30 \mathrm{MIN}$ & 7.8846 & 0.3258 & 7.9 & 0.3051 & 0.08558 \\
\hline Positioning & 2.5 & 0.7671 & 2.4666 & 0.5074 & 0.8465 \\
\hline
\end{tabular}

Among the patients undergoing spinal anaesthesia in fracture femur surgery, there was no statistically significant difference in Numerical rating scale (NRS) for pain between the groups. Group A at baseline $($ mean $=8.1538, \mathrm{SD}=0.464)$, after 30 minutes (mean=7.8846, $\mathrm{SD}=0.3258)$, during positioning (mean $=2.5, \mathrm{SD}=0.7671$ ) and Group $\mathrm{B}$ at baseline $($ mean $=8.1, \mathrm{SD}=0.4806)$, after 30 minutes (mean=7.9, $\mathrm{SD}=0.3051)$, during positioning (mean $=2.466, \mathrm{SD}=0.0 .5074)$ with a $\mathrm{p}$ value of $>0.05$ as per unpaired t test. Therefore we fail to reject the null hypothesis that there is no difference in the numerical rating scale between the intervention groups.

\section{Table 2: QUALITY OF POSITIONING}

\begin{tabular}{|l|l|l|l|l|}
\hline \multirow{2}{*}{ QUALITY OF POSITIONING } & \multicolumn{3}{|l|}{ GROUP A } & \multicolumn{2}{l|}{ GROUP B } \\
\cline { 2 - 5 } & No & $\%$ & No & $\%$ \\
\hline 0 : NOT SATISFACTORY & 1 & 3.3 & 1 & 3.3 \\
\hline 1 : SATISFACTORY & 11 & 36.7 & 10 & 33.3 \\
\hline 2 : GOOD & 15 & 50 & 16 & 53.4 \\
\hline 3 : OPTIMAL & 3 & 10 & 3 & 10 \\
\hline
\end{tabular}

Among the patients undergoing spinal anaesthesia in fracture femur surgery, $3.3 \%$ of patients of both the groups had not satisfactory positioning, $36.7 \%$ of Group A and $33.3 \%$ of Group B had satisfactory positioning, $50 \%$ of Group A and $53.4 \%$ of Group B patients had good positioning, $10 \%$ of patients of both groups had optimal positioning. Therefore the positioning of the patients for spinal anaesthesia is comparable and is no significant difference is seen among both the groups.

Table 3: TIME TO PERFORM SUB ARACHANOID BLOCK

\begin{tabular}{|l|l|l|}
\hline & GROUPA & GROUP B \\
\hline N & 30 & 30 \\
\hline MEAN & 5.846 & 5.183 \\
\hline SD & 2.538 & 2.155 \\
\hline P VALUE & 0.2801 & \\
\hline
\end{tabular}

Among the patients undergoing spinal anaesthesia in fracture femur surgery, there was no statistically significant difference in the time taken to perform the sub arachanoid block between Group A (mean $=5.846, \mathrm{SD}=2.538)$ and Group $\mathrm{B}($ mean $=5.183, \mathrm{SD}=2.155)$ with a $p$ value of $0.2801(\mathrm{p}>0.05)$ as per unpaired t test. Therefore we fail to reject the null hypothesis that there is no difference in time taken to perform the sub arachanoid block between the intervention groups.

Table 4: ATTEMPTS TAKEN TO PERFORM SUB ARACHANOID BLOCK

\begin{tabular}{|l|l|l|l|l|}
\hline \multirow{2}{*}{ ATTEMPT } & \multicolumn{3}{|l|}{ GROUP A } & \multicolumn{2}{l|}{ GROUP B } \\
\cline { 2 - 5 } & No & $\%$ & No & $\%$ \\
\hline 1 & 16 & 53.4 & 16 & 53.4 \\
\hline 2 & 11 & 36.6 & 10 & 33.3 \\
\hline 3 & 3 & 10 & 4 & 13.3 \\
\hline TOTAL & 30 & 100 & 30 & 100 \\
\hline
\end{tabular}

Among the patients undergoing spinal anaesthesia in fracture femur surgery, there was no significant difference in the no. of attempts taken to perform the sub arachanoid block between the 2 groups. Group A first, second and third attempt are $53.4 \%, 36.6 \%$ and $10 \%$ respectively and Group B first, second and third attempt are 53.4\%, 33.3\% and $13.3 \%$ respectively.

\section{DISCUSSION:}

This prospective, randomized, double blinded study, was done to evaluate the improvement in positioning for SAB with prior administration of inj paracetamol to fascia iliaca compartment block under ultrasound guidance with ropivacaine,. 60 patients satisfying the inclusion criteria were chosen and divided into two groups of thirty each. Group A patients received ultrasound guided fascia iliaca compartment block with $30 \mathrm{ml}$ of $0.5 \%$ Ropivacaine with 30 min prior administration of $100 \mathrm{ml} \mathrm{NS} \mathrm{I.V} \mathrm{while} \mathrm{Group} \mathrm{B} \mathrm{patients} \mathrm{received}$ fascia iliaca compartment block with $30 \mathrm{ml}$ of $0.5 \%$ of Ropivacaine with 30min prior administration of inj Paracetamol $1 \mathrm{~g}$ (i.v).
The mean age was in $56.566 \pm 11.536 \mathrm{FICB}$ with NS group and 59.615 \pm 12.202 in FICB with Paracetamol group. The sex distribution in FICB with NS group was 14 males and 16 females while in FICB with Paracetamol group, there were 16 males and 14 females. The mean BMI in FICB group was $21.134 \pm 2.819$ while in FICB with Paracetamol group it was $22.2 \pm 3.622$. Thus both the groups were comparable and no significant difference is seen in demographic profile in terms of age, sex and BMI distribution as the $\mathrm{P}$ value was not significant $(\mathrm{P}>0.05)$. The duration since fracture to surgery was FICB $6.731 \pm 9.37$ days and FICB with Paracetamol 6.933 \pm 10.194 was also insignificant $(\mathrm{P}>0.05)$

The Numerical Rating Scale score during positioning was $2.5 \pm 0.7071$ in FICB group and 2.466 \pm 0.507 in FICB with Paracetamol group and was statistically insignificant with a $\mathrm{P}$ value of 0.8465 . It shows that fascia iliaca compartment block provides better analgesia for patient positioning in fracture femur surgeries and addition of Paracetamol i.v did not have significant change in the scores.

The quality of patient positioning in FICB group with a mean of $2.115 \pm 0.816$ and FICB with Paracetamol group which had a mean of $2.2 \pm 0.846$. The difference was statistically insignificant with a $P$ value of 0.6935 ( $p>0.005)$. It means that fascia iliaca compartment block provides better quality of patient positioning for spinal anaesthesia but addition of Paracetamol i.v did not have significant effect.

The time taken to perform subarachnoid block (time from beginning of positioning to end of spinal) in FICB group 5.846 \pm 02.538 compared to FICB with Paracetamol group $5.183 \pm 2.155$. It was statistically insignificant with a Pvalue of 0.2801 .

The difference in hemodynamics and $\mathrm{SpO} 2$ between the 2 groups was statistically insignificant $(\mathrm{P}>0.005)$

There were no complications of block like infection, block failure, vascular puncture, nerve damage or systemic toxicity of local anaesthetics in both groups.

In this study, Fascia Iliaca Compartment Block provided superior analgesia and addition of Paracetamol (i.v) did not improve analgesia significantly nor positioning comfort during spinal anaesthesia. The quality of patient positioning also did not show any significant difference with addition of Paracetamol (i.v). There was also no significant difference in either time taken to perform subarachnoid block or the no. of attempts taken to perform subarachanoid block as with other studies ${ }^{9,10}$

\section{CONCLUSION:}

In this study the pre administration of inj Paracetamol $1 \mathrm{~g}$ i.v to Fascia Iliaca Compartment Block did not produce any additional improvement in analgesia and positioning for central neuraxial blockade in patients posted for surgical repair of fracture femur.

\section{REFERENCES:}

1. Richard.F.Kyle, Fracture of the proximal part of the femur, Journal of Bone and Join Surgery(JBJS) 1994

2. Sandrine Lopez, Thierry Gros, Nathalie Bernard, Christian Plasse and Xavie Capdevila. Fascia Iliaca Compartment Block for Femoral Bone Fractures in Prehospital Care. Regional Anesthesia and Pain Medicine, Vol 28, No 3 (May-June), 2003: pp 203-207.

3. Seunguk Bang, Jihyun Chung, Jaejung Jeong, Hahyeon Bak, Dongju Kim. Efficacy of ultrasound-guided fascia iliaca compartment block after hip hemiarthroplasty: A prospective, randomized trial. Medicine (2016) 95:39(e5018)

4. Morrison RS, Magaziner J, Gilbert M, et al. Relationship between pain and opioid analgesics on the development of delirium following hip fracture. J Gerontol A Biol Sc Med Sci 2003;58:76-81.

5. M. Sandby Thomas, G. Sullivan and J. E. Hall .A national survey into the peri-operative anaesthetic management of patients presenting for surgical correction of a fractured neck of femur: Journal compilation 2008 The Association of Anaesthetists of Great Britain and Ireland

6. Dalens B, Tanguy A, Vanneuville G. Lumbar plexus blocks and lumbar plexus nerve blocks. Anesth Analg 1989:69:852-4

7. X. Capdevila Biboulet, M. Bouregba, Y. Barthelet, J. Rubenovitch, and F. d'Athis Comparison of the Three-in-One and Fascia lliaca Compartment Blocks in Adults: Clinical and Radiographic Analysis. Anesth Analg 1998;86:103944 1039

8. Sandrine Lopez, Thierry Gros, Nathalie Bernard,Christian Plasse, and Xavier Capdevila Fascia Iliaca Compartment Block for Femoral Bone Fractures in Prehospital Care. Regional Anesthesia and Pain Medicine, Vol 28, No 3 (May-June), 2003: pp 203-207.

9. Bonica JJ. Improtance of effective pain control. Acta Anaeshesiol Scand 1987;31(Supp 85):1-16.

10. Morrison RS, Magaziner J, Gilbert M, et al. Relationship between pain and opioid analgesics on the development of delirium following hip fracture. J Gerontol A Biol Sci Med Sci 2003;58:76-81. 